\title{
Integrated Wavelength-Tunable Light Source for Optical Gas Sensing Systems
}

\author{
Bin Li, Qi-Xin He, Hui-Fang Liu, and Yi-Ding Wang \\ State Key Laboratory on Integrated Optoelectronics, College of Electronic Science and Engineering, Jilin University, \\ Changchun 130012, China
}

Correspondence should be addressed to Yi-Ding Wang; wangyiding48@hotmail.com

Received 30 September 2015; Accepted 25 October 2015

Academic Editor: Stefan Wabnitz

Copyright (C) 2015 Bin Li et al. This is an open access article distributed under the Creative Commons Attribution License, which permits unrestricted use, distribution, and reproduction in any medium, provided the original work is properly cited.

A compact instrument consisting of a distributed feedback laser (DFB) at $1.65 \mu \mathrm{m}$ was developed as a light source for gas sensing systems using tunable diode laser absorption spectroscopy (TDLAS) technique. The wavelength of laser is tuned by adjusting the laser working temperature and injection current, which are performed by self-developed temperature controller and current modulator respectively. Stability test shows the fluctuation of the laser temperature is within the range of $\pm 0.02^{\circ} \mathrm{C}$. For gas detection experiments, the wavelength is tuned around the gas absorption line by adjusting laser temperature and is then shifted periodically to scan across the absorption line by the laser current modulator, which generates a $10 \mathrm{~Hz}$ saw wave signal. In addition, the current modulator is able to generate sine wave signal for gas sensing systems using wavelength modulation spectroscopy (WMS) technique involving extraction of harmonic signals. The spectrum test proves good stability that the spectrum was measured 6 times every 10 minutes at the constant temperature and current condition. This standalone instrument can be applied as a light source for detection systems of different gases by integrating lasers at corresponding wavelength.

\section{Introduction}

Monitoring specific gases, such as methane $\left(\mathrm{CH}_{4}\right)$, carbon monoxide $(\mathrm{CO})$, and carbon dioxide $\left(\mathrm{CO}_{2}\right)$, are indispensable in many fields [1-4]. Methane, for instance, is not only the second significant greenhouse gas [5], but also a hazard gas in coalmines for causing explosion [6]. Existing gas sensing technologies generally include optical absorption, electrochemical, semiconductor, and catalyst combustion [7-10]. Optical absorption technique generally has the advantages including sensitive species-specific detection, quick response, and nonintrusive nature [11]. TDLAS is an efficient spectroscopic tool for gas detection. It offers a promising solution to gas concentration and temperature detections based on a significant property of tunable diode laser; that is, the wavelength can be shifted by changing the laser's driving conditions. In TDLAS systems, DFB lasers and quantum cascaded (QC) lasers are frequently applied as light sources for excellent spectroscopic characteristics like wide tuning range and narrow spectral line width $[12,13]$. DFB laser, which is a type of diode laser, is widely deployed in many applications including the near-infrared (NIR) gas sensing systems. Compared to QC lasers, DFB lasers are normally coupled with optical fibers that are suitable for long-distance detection and multipoint detection applications. DFB lasers also normally have lower cost and power consumption than QC lasers. Along with the lasers, commercial instruments including temperature controller, digital signal generator, and laser drivers are normally implemented to effectively control the laser wavelength [14-16]. However, the drawbacks of the commercial instruments are known as expensive, complex, and large-size. Therefore, it is essential to develop a specialized DFB driving instrument as a standalone light source to satisfy the requirements of experiments and overcome the problems.

In this paper, a self-developed instrument integrating a DFB laser at $1.65 \mu \mathrm{m}$ is proposed. Inside the instrument, the laser temperature controller and current modulator are developed and integrated to control the DFB laser wavelength. Compared to the commercial apparatuses, this standalone instrument has a much lower cost and is easy to operate. 
Experiments were carried out as well as results suggesting good performance of the instrument. The rest of this paper is organized as below. First in Section 2, detection theory and related formulation are briefly summarized. In Section 3, both design and integration details are proposed with performance test for each module. Furthermore in Section 4, the modulation performances of laser spectrum are derived through experiments. Finally the conclusions are in Section 5.

\section{Theory}

The attenuation of spectral intensity, which is caused by absorption of gas molecules, is governed by the Beer-Lambert law that is shown in (1) where $I(v)$ is the transmitted intensity, $I_{0}(v)$ is the incident intensity, $L$ is the optical path length, and $C$ is the gas concentration. The laser emitting intensity at center wavelength, which is expressed as $I_{0}$ in (2), is constant by fixed driving current and working temperature. WMS technique and direct absorption spectroscopy (DAS) are two variations of TDLAS [17]. In WMS systems, superior to DAS for achieving a better signal-to-noise ratio (SNR), the laser is additionally driven by a sine wave signal in the kilohertz range and a saw wave signal in a much lower frequency. The two signals are expressed in (3) and (4). Thus, the emitting intensity is derived in (2) as $I(t)$ where $m$ is the modulation factor. In this instrument, the adjustable frequencies of the sine wave and saw wave signals are set as $5 \mathrm{kHz}$ and $10 \mathrm{~Hz}$, respectively:

$$
\begin{aligned}
\frac{I(v)}{I_{0}(v)} & =\exp [-\alpha(v, t) L C], \\
I(t) & =I_{0}\left[1+m u_{\text {saw }}(t)+m u_{\text {sin }}(t)\right], \\
u_{\text {saw }}(t) & =a+\frac{a}{T_{\text {saw }}}\left(t-T_{\text {saw }}\right), \\
u_{\text {sin }}(t) & =b \sin \left(\omega_{\text {sin }} t\right) .
\end{aligned}
$$

\section{Design Details}

3.1. System Structure. System structure of the developed instrument is shown in Figure 1. The main controller of the instrument is a digital signal processor (DSP) with features including high-performance 32-bit central processing unit (CPU), enhanced control peripherals, and sufficient on-chip memories. The optimized program is loaded on the on-chip memories and there are no external memory chips utilized. The main controller is able to govern the three sections of the instrument which are temperature controller, current modulator, and user interface such as the liquid crystal display (LCD) screen and input/output ports. In order to shrink instrument size and ensure good performance, modules including the main controller, the temperature, and the current modulator are integrated on one 4-layer printed circuit board (PCB). The installing socket of the DFB laser is also located on the board to shorten the essential connecting lines. Under the control of the main controller, the temperature controller circuits are responsible for adjusting and maintaining the laser working temperature. Meanwhile,

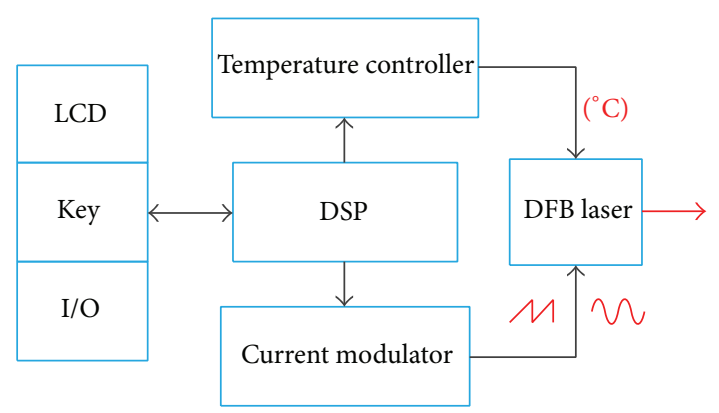

FIGURE 1: System structure of the designed DFB driving instrument.

the current modulator is driving the laser in the range of 40-80 mA. It supplies saw wave signal for gas absorption applications and additionally sine wave signal for harmonic extraction applications. Users can set the temperature value, current mode, and signal ON/OFF by the corresponding keys embedded on the front penal of the instrument and the real time conditions of the parameters are shown on the LCD. The optical output of the instrument is through a FC/APC port which is on the back penal.

3.2. Temperature Controller. Temperature control of DFB laser is crucial for gas absorption experiments and other precisely spectroscopic applications. This is due to the fact that the wavelength of the DFB laser is largely affected by the working temperature. When the temperature varies, the reflection rate of optical grating changes and the output wavelength shifts. In experiments of gas detections, the laser temperature should be set precisely to tune the wavelength around the gas absorption line and be maintained stably to avoid wavelength shifting. In this instrument, the laser temperature can be adjusted with the step of $1^{\circ} \mathrm{C}$ or $0.05^{\circ} \mathrm{C}$ depending on specific conditions.

The integrated DFB laser is provided by Institute of Semiconductors, Chinese Academy of Sciences (ISCAS). The center wavelength of the laser is around $1.65 \mu \mathrm{m}$ which is suitable for methane detection. It is pigtailed with a $30 \mathrm{~cm}$ optical fiber and is 14-pin butterfly packaged. A thermoelectric cooler (TEC) is integrated inside the laser for temperature controlling with a negative temperature coefficient (NTC) thermistor. The TEC is controlled by driving current in two directions for cooling and heating separately. This is based on the Peltier effect. The NTC thermistor is used for sensing laser temperature. Its resistor value changes with the temperature variation. The laser temperature is maintained by an analog proportion-integral-differential (PID) control loop circuit.

Experiments were carried out to evaluate the temperature control performances. First of all, the stability test was performed to investigate the temperature fluctuation in the room temperature environment. The laser temperature was set at $25.65^{\circ} \mathrm{C}$ and the voltage signal of temperature was sampled every 30 seconds by ADC module. During a period of 20 hours, the result of stability test is shown in Figure 2. The fluctuation of the temperature is within the range of $\pm 0.02^{\circ} \mathrm{C}$ as shown in the figure. This suggests good stability of 


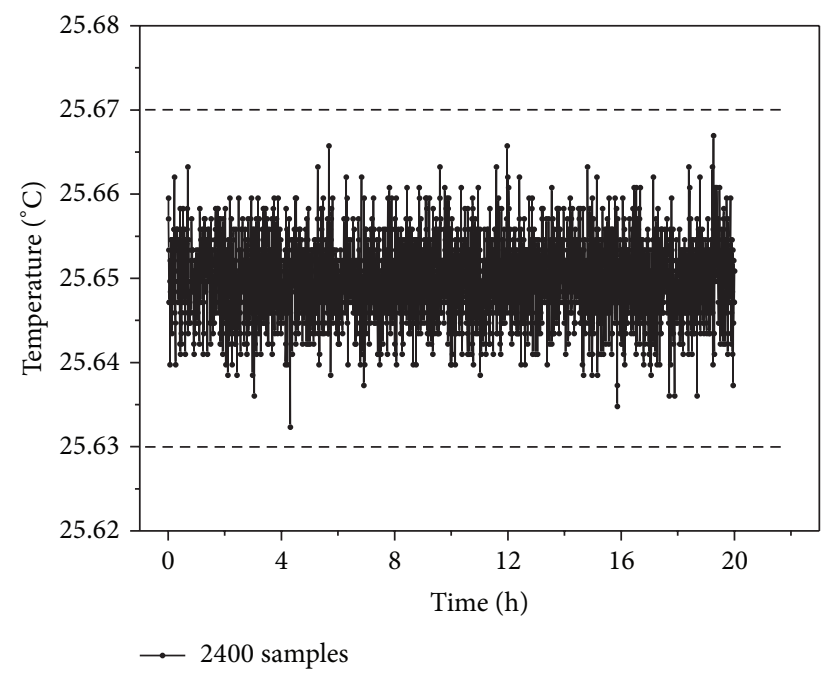

FIGURE 2: Stability test of the laser temperature controller.

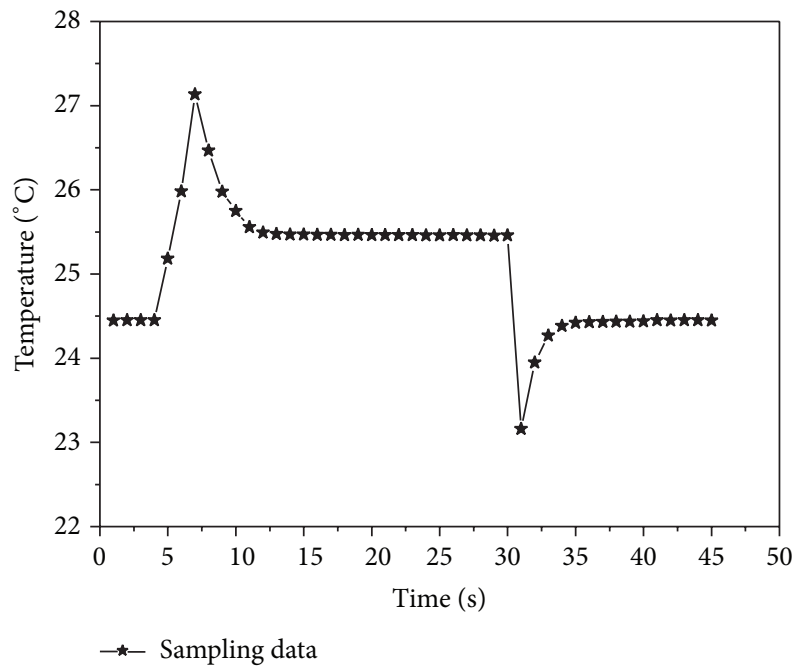

FIGURE 3: Response time measurement while changing the laser temperature.

the temperature controlling performance. Then, the response time test was performed to evaluate the PID control loop circuit. The temperature was measured while increasing and decreasing $1^{\circ} \mathrm{C}$ and the result is shown in Figure 3 . The laser temperature is effectively adjusted to the target value in 10 seconds which is acceptable for gas absorption experiments.

3.3. Current Modulator. DFB laser is generally modulated by a low frequency saw wave signal and a high frequency sine wave signal together in gas detection systems based on TDLAS-WMS technique. The laser is driven by constant current in this instrument. There is a digital-to-analog converter (DAC, AD5060) deployed for generating the $10 \mathrm{~Hz}$ saw wave signal. Simultaneously, a direct digital synthesizer (DDS, AD9851) is applied to generate a $5 \mathrm{kHz}$ sine wave signal for harmonic extractions. The frequency of the signals can be changed by modifying the program. Then, the two signals are combined together by an addition circuit to be superimposed on the laser. In addition, the DDS chip also generates a square wave signal which has the same frequency and phase as the sine signal. It is the reference signal for $1 f$ and $2 f$ harmonic extractions.

The optical output of the laser is equally divided into two beams after the current modulation. One beam passes through the gas cell for absorption and the other beam is linked to an optical attenuator as reference signal. The two optical signals are then converted to electrical signals by two InGaAs photodiodes with the same optical-to-electrical responsibility of $1.1 \mathrm{~A} / \mathrm{W}$. The voltage signals are measured by an oscilloscope (Tektronix, TDS3032C) as shown in Figure 4. The reference signal must be adjusted to match the absorption signal by changing the feedback resistor value. The saw wave signals are shown in Figure 4(a). Then, the sine wave signal is superimposed on the laser and shown in Figure 4(b). Photograph of the final integrated instrument is shown in Figure 5. On the front panel, there is LCD screen with the resolution of $320 \times 240$ and functional keys including buttons of temperature modulation, current modulation, and ON/OFF signals. The optical output port is located on the back panel.

\section{Experimental Results}

Experiments were carried out to evaluate the spectroscopic performance of the instrument. Firstly, spectrum was measured by adjusting the laser temperature while other conditions were maintained. The injection current was set as $70 \mathrm{~mA}$. The result of the measurement is shown in Figure 6. The temperature was adjusted with a step of $1^{\circ} \mathrm{C}$. As shown in the figure, the center wavelength of the laser is linearly shifted with the temperature variation. This result proves that the wavelength is effectively tuned by adjusting the laser temperature. Therefore, the output wavelength of the laser can be tuned to approach the gas absorption line by adjusting the laser temperature.

Then, the driving current of the laser was changed and the corresponding spectrum was measured. As shown in Figure 7, current modulation causes wavelength shifting while the temperature is maintained at $25^{\circ} \mathrm{C}$. The current is increased from $40 \mathrm{~mA}$ to $80 \mathrm{~mA}$ with the step of $10 \mathrm{~mA}$. Meanwhile, the optical intensity is also largely affected by tuning the driving current. While the driving current increases, the optical intensity rises proportionally. In gas sensing experiments, the wavelength of the laser is tuned close to the absorption line by adjusting laser temperature to a specific value firstly. Then, the laser temperature is fixed at this point during the experiment. The driving current is periodically changed in order to tune the wavelength. In this way, the wavelength periodically scans across the gas absorption line for the absorption phenomenon. The current is modulated 2000 times per second which is performed by a DSP timer in this instrument.

The stability test was performed in order to evaluate the instrument performance. During a period of 1 hour, the spectrum was measured six times per 10 minutes as shown in Figure 8 . The temperature was fixed at $30^{\circ} \mathrm{C}$ and the driving 


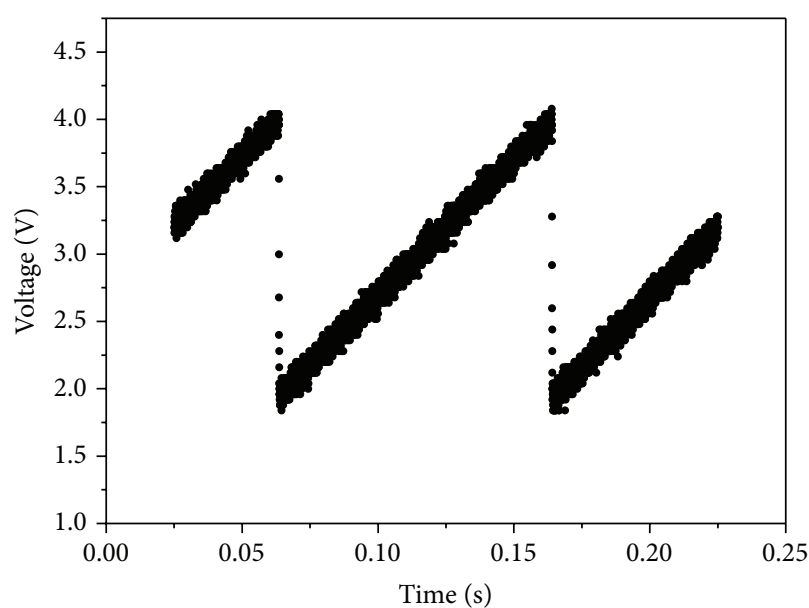

- Samples

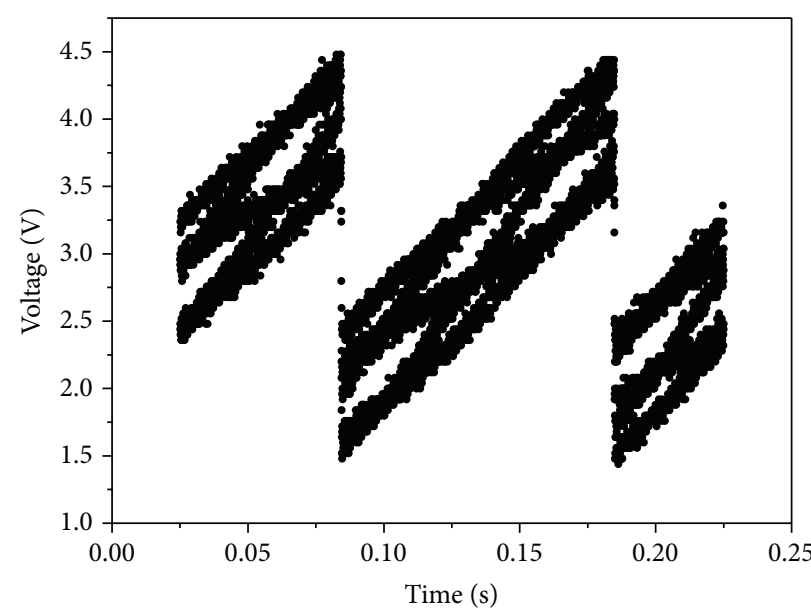

- Samples

(a)

(b)

FIGURE 4: The measured signals of the photodiodes: (a) laser is driven by the saw wave signal and (b) laser is driven by the saw wave signal and sine wave signal.

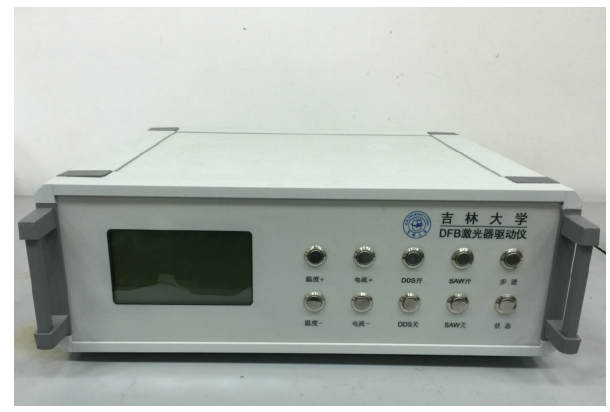

FIGURE 5: Photograph of the integrated DFB driving instrument.

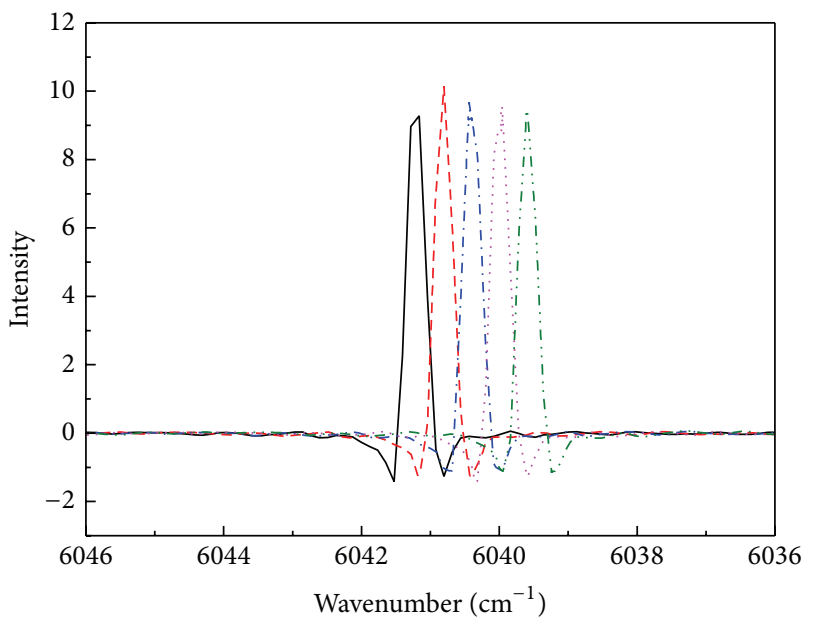

Laser driving current: $70 \mathrm{~mA}$

$$
\begin{aligned}
& -28^{\circ} \mathrm{C} \quad \cdots \cdots 31^{\circ} \mathrm{C} \\
& --29^{\circ} \mathrm{C} \quad-\cdots 32^{\circ} \mathrm{C} \\
& \text {-. . } 30^{\circ} \mathrm{C}
\end{aligned}
$$

FIGURE 6: Spectrum measurement with laser temperature variety from $28^{\circ} \mathrm{C}$ to $32^{\circ} \mathrm{C}$.

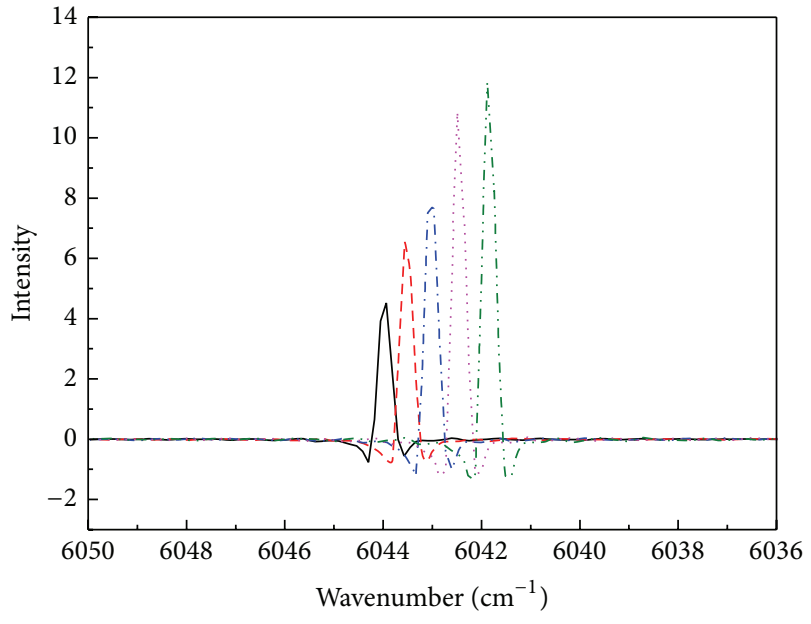

Temperature: $25^{\circ} \mathrm{C}$

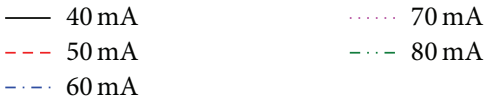

FIGURE 7: Spectrum measurement with the laser driving current increasing from $40 \mathrm{~mA}$ to $80 \mathrm{~mA}$.

current was set at $80 \mathrm{~mA}$. As shown in the figure, the measured spectrum was stable and the center wavelength was not shifting during the test. Therefore, the result proves good stability of the spectroscopic performance of the instrument.

\section{Conclusion}

A standalone instrument was designed and developed for gas sensing systems. A distributed feedback laser at $1.65 \mu \mathrm{m}$, which is for methane detection, is integrated in this instrument. Laser wavelength can be tuned by self-developed laser 


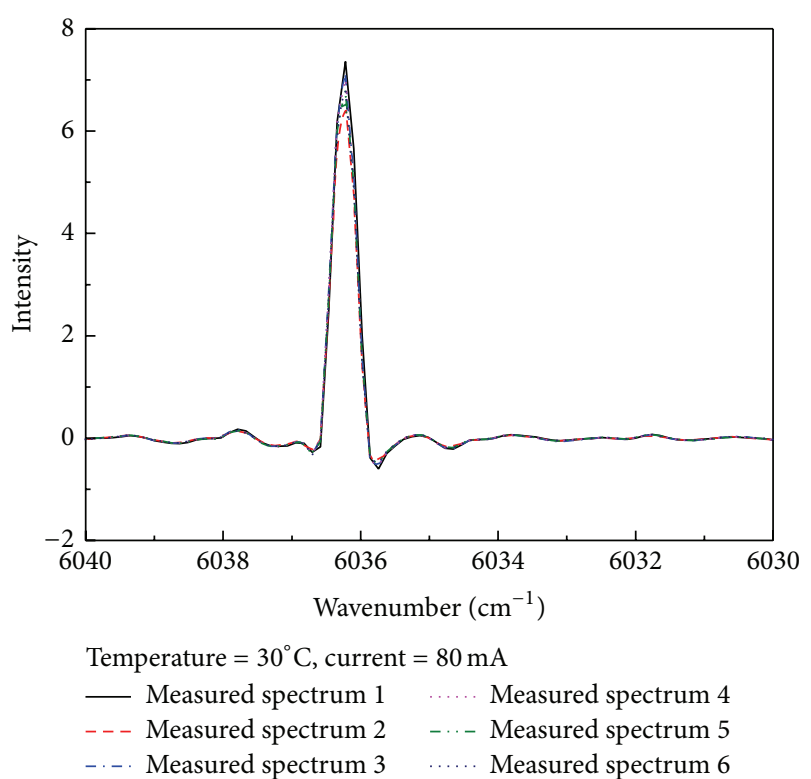

FIGURE 8: Stability test of the laser center wavelength under constant laser temperature and driving current.

temperature controller and current modulator. Experiment results prove good stability of the laser temperature and injection current. The fluctuation of the temperature is determined within the range of $\pm 0.02^{\circ} \mathrm{C}$ and the spectrum was stable during a one-hour measurement. The current modulator is able to generate low frequency saw wave signal and high frequency sine wave signal for WMS systems. Instead of using commercial devices such as laser temperature controller and signal generator, the self-integrated instrument proves acceptable performance as a light source for gas detection systems. Besides, it has a smaller size and much lower cost than the commercial devices. By integrating lasers at other wavelengths, the instrument can be applied as a light source in sensing systems for the corresponding trace gases.

\section{Conflict of Interests}

The authors declare that there is no conflict of interests regarding the publication of this paper.

\section{Acknowledgments}

The authors wish to express their gratitude to the National Key Technology R\&D Program of China (nos. 2013BAK06B04 and 2014BAD08B03), the National Natural Science Foundation of China (nos. 61307124 and 11404129), the Science and Technology Department of Jilin Province of China (nos. 20120707 and 20140307014SF), the Changchun Municipal Science and Technology Bureau (nos. 11GH01 and 14KG022), and the State Key Laboratory of Integrated Optoelectronics, Jilin University (no. IOSKL2012ZZ12), for their generous support of this work.

\section{References}

[1] N. Rajabbeigi, B. Elyassi, A. A. Khodadadi, S. Mohajerzadeh, Y. Mortazavi, and M. Sahimi, "Oxygen sensor with solid-state $\mathrm{CeO}_{2}-\mathrm{ZrO}_{2}-\mathrm{TiO}_{2}$ reference," Sensors and Actuators B: Chemical, vol. 108, no. 1-2, pp. 341-345, 2005.

[2] Q. C. Liang, D. Li, S. Gao et al., "Room-temperature $\mathrm{NH}_{3}$ sensors with high sensitivity and short response/recovery times," Chinese Science Bulletin, vol. 59, no. 4, pp. 447-451, 2014.

[3] H. Dacres and R. Narayanaswamy, "A new optical sensing reaction for nitric oxide," Sensors and Actuators B: Chemical, vol. 90, no. 1-3, pp. 222-229, 2003.

[4] S. Svanberg, "Chemical sensing with laser spectroscopy," Sensors and Actuators B: Chemical, vol. 33, no. 1-3, pp. 1-4, 1996.

[5] C. Chen, R. W. Newcomb, and Y. Wang, "A trace methane gas sensor using mid-infrared quantum cascaded laser at $7.5 \mu \mathrm{m}$," Applied Physics B: Lasers and Optics, vol. 113, no. 4, pp. 491-501, 2013.

[6] B. S. Nie, C. Wang, J. Q. Meng, F. Xue, and L. C. Dai, "Laboratory investigation into fractal characteristics of methane explosion flame," Process Safety Progress, vol. 34, no. 3, pp. 244-249, 2015.

[7] M. Choudhary, V. N. Mishra, and R. Dwivedi, "Solid-state reaction synthesized $\mathrm{Pd}$-doped tin oxide thick film sensor for detection of $\mathrm{H}_{2}, \mathrm{CO}, \mathrm{LPG}$ and $\mathrm{CH}_{4}$," Journal of Materials Science: Materials in Electronics, vol. 24, no. 8, pp. 2824-2832, 2013.

[8] Y. C. Cao, N. P. Sanchez, W. Z. Jiang et al., "Simultaneous atmospheric nitrous oxide, methane and water vapor detection with a single continuous wave quantum cascade laser," Optics Express, vol. 23, no. 3, pp. 2121-2132, 2014.

[9] M. Wolff, S. Rhein, H. Bruhns, L. Nähle, M. Fischer, and J. Koeth, "Photoacoustic methane detection using a novel DFBtype diode laser at $3.3 \mu \mathrm{m}$," Sensors and Actuators B: Chemical, vol. 187, pp. 574-577, 2013.

[10] Q. Wang, J. Chang, W. Wei, C. G. Zhu, and C. B. Tian, "Dual-beam wavelength modulation spectroscopy for sensitive detection for water vapor," Applied Physics B: Lasers and Optics, vol. 117, no. 4, pp. 1015-1023, 2014.

[11] R. Sur, K. Sun, J. B. Jeffries et al., "TDLAS-based sensors for in situ measurement of syngas composition in a pressurized, oxygen-blown, entrained flow coal gasifier," Applied Physics B: Lasers and Optics, vol. 116, no. 1, pp. 33-42, 2014.

[12] J. Shemshad, "Analysis of inaccuracy induced by intensity variation of a DFB laser in fibre optic multipoint $2 \mathrm{f}-\mathrm{WMS}$ measurements of methane near $1666 \mathrm{~nm}$," Sensors and Actuators A: Physical, vol. 222, pp. 96-101, 2015.

[13] Y. J. Zhao, Q. P. Wang, J. Chang et al., "Suppression of the intensity noise in distributed feedback fiber lasers by selfinjection locking," Laser Physics Letters, vol. 9, no. 10, pp. 739743,2012

[14] S. Neethu, R. Verma, S. S. Kamble, J. K. Radhakrishnan, P. P. Krishnapur, and V. C. Padaki, "Validation of wavelength modulation spectroscopy techniques for oxygen concentration measurement," Sensors and Actuators B: Chemical, vol. 192, pp. 70-76, 2014.

[15] R. Sur, K. Sun, J. B. Jeffries, J. G. Socha, and R. K. Hanson, "Scanned-wavelength-modulation-spectroscopy sensor for $\mathrm{CO}, \mathrm{CO}_{2}, \mathrm{CH}_{4}$ and $\mathrm{H}_{2} \mathrm{O}$ in a high-pressure engineeringscale transport-reactor coal gasifier," Fuel, vol. 150, pp. 102-111, 2015. 
[16] D. Mondelain, T. Sala, S. Kassi, D. Romanini, M. Marangoni, and A. Campargue, "Broadband and highly sensitive combassisted cavity ring down spectroscopy of CO near $1.57 \mu \mathrm{m}$ with sub-MHz frequency accuracy," Journal of Quantitative Spectroscopy \& Radiative Transfer, vol. 154, pp. 35-43, 2015.

[17] B. Lins, P. Zinn, R. Engelbrecht, and B. Schmauss, "Simulationbased comparison of noise effects in wavelength modulation spectroscopy and direct absorption TDLAS," Applied Physics B: Lasers and Optics, vol. 100, no. 2, pp. 367-376, 2010. 

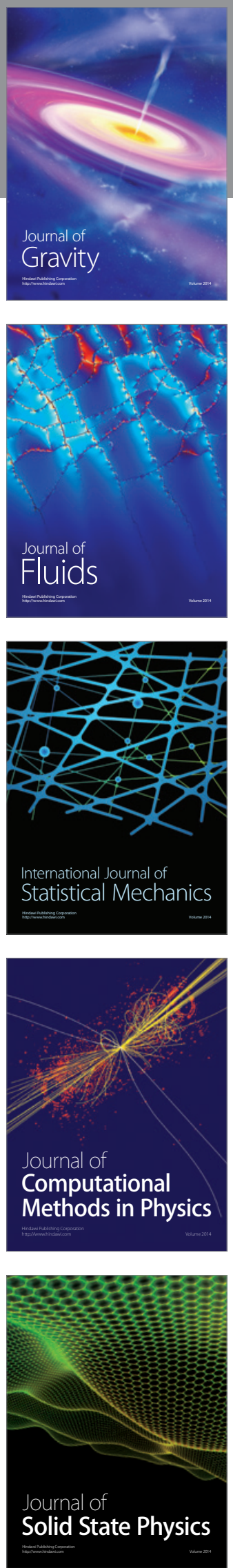

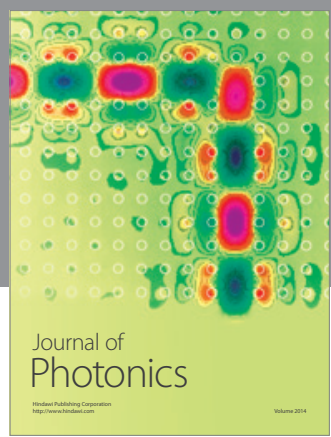

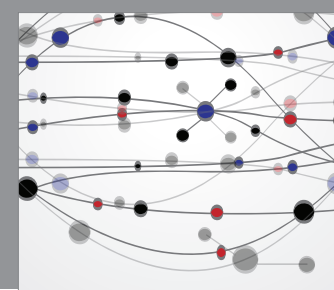

The Scientific World Journal

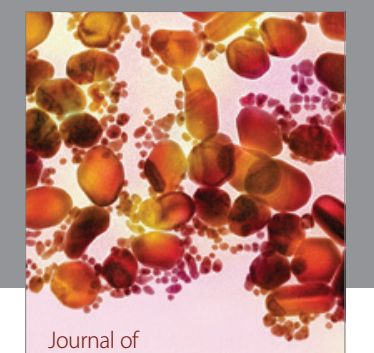

Soft Matter
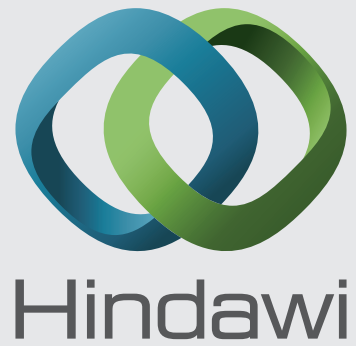

Submit your manuscripts at

http://www.hindawi.com
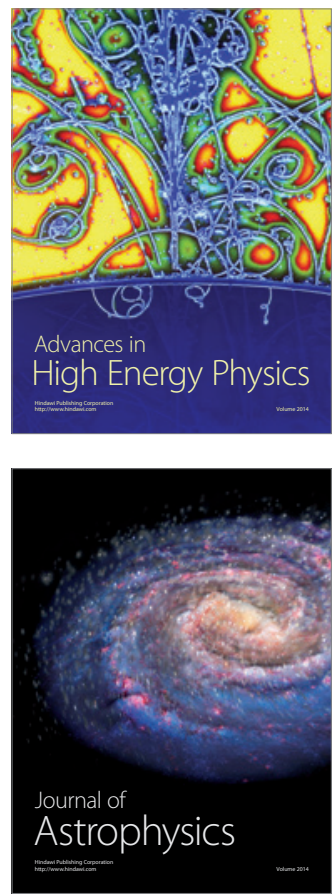
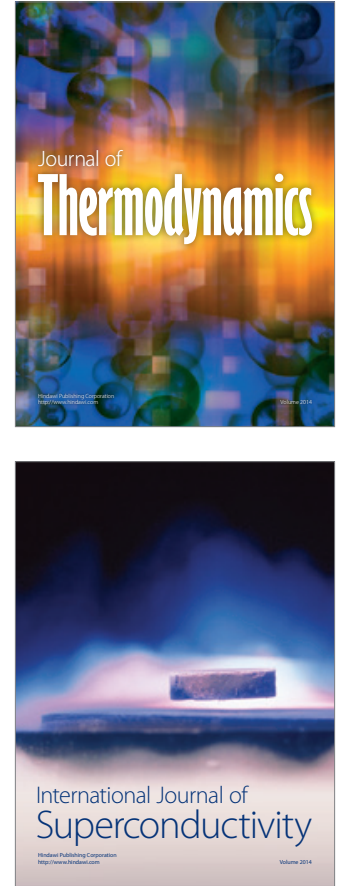
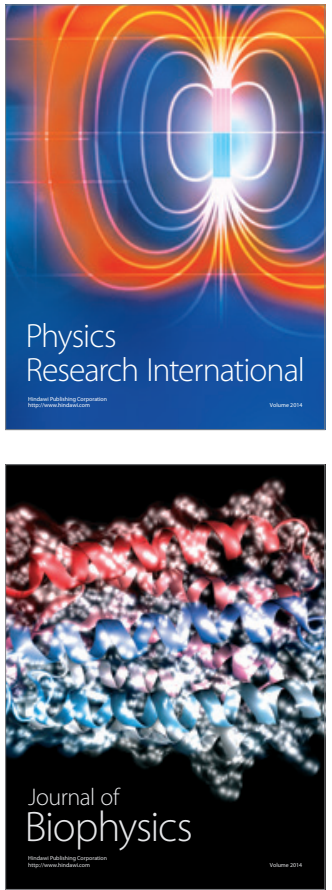
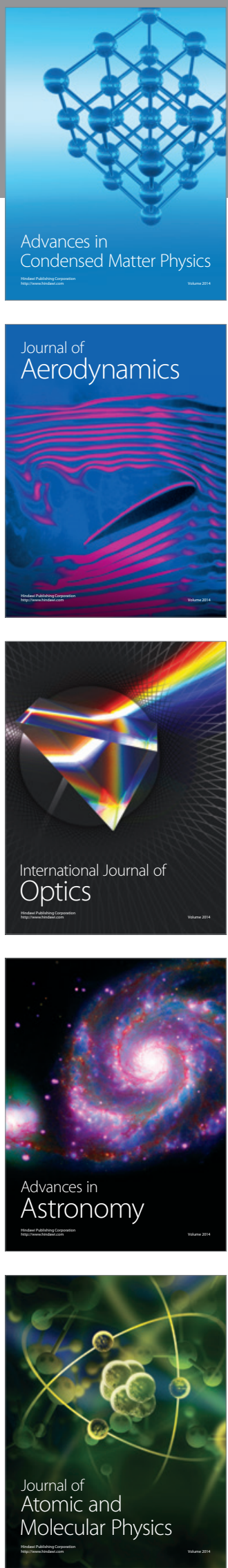